\title{
Substructures and galaxy orientations in clusters II. Cluster Abell 14
}

\author{
F. W. Baier ${ }^{1}$, W. Godłowski ${ }^{2}$, and H. T. MacGillivray ${ }^{3}$ \\ 1 Institute for Communication and Structure Research, G.-Hauptmann-Str. 16, 14558 Bergholz-Rehbrücke, Germany \\ 2 Jagellonian University, Astronomical Observatory, ul. Orla 171, 30-244 Krakow, Poland \\ 3 Royal Observatory, Blackford Hill, Edinburgh, EH9 3HJ, Scotland, UK
}

Received 22 December 2000 / Accepted 27 February 2003

\begin{abstract}
We present an investigation of the Abell 14 galaxy cluster, including a discussion of subclustering, some aspects concerning its evolution, as well as an analysis of galaxy orientations in the cluster region. We confirm the previous results showing evidence for subclustering in this cluster obtained from studies of galaxy distributions. In this light, our aim has been to examine non-random effects in the galaxy orientations in rich clusters, with particular regard to the presence of subclustering. Our results do provide evidence for non-random alignment of the galaxies in Abell 14 and confirm the presence of subclustering within this cluster. However, the direction of the alignment is different from that (we) found for Abell 754 (Godłowski et al. 1998). We continue our investigations by studying other clusters in a similar manner. The results will be published in the next paper.
\end{abstract}

Key words. methods: statistical - galaxies: clusters: general - galaxies: clusters: individual: Abell 14

\section{Introduction}

Originally, galaxy clusters were characterized by the BM types I, II, and III (Bautz \& Morgan 1970) or by the RS types cD, B, L, F, and I (Struble \& Rood 1987). The existence of optical and X-ray substructures in a considerable number of clusters became evident soon after (e.g. Baier \& Mai 1978a,b; Baier 1979a,b,c; Baier 1980a,b; Geller \& Beers 1982; Dressler \& Shectman 1988; Jones \& Forman 1992; Mohr et al. 1995). In the past there were quite different results for the percentage of galaxy clusters with substructures: Geller \& Beers (1982) found it to be $40 \%$ and Baier (1983) estimated it at $\sim 70$ $80 \%$ from his optical investigations. Further results are: $35 \%$ (Webster et al. 1988), 30\% (Jones \& Forman 1990; Briel et al. 1991), 26\% (Rhee et al. 1991), 45\% (Jones \& Forman 1992), $85 \%$ (Bird 1994). Flin \& Krywult (2000) argued that the determination of the percentage of galaxy cluster with subclustering strongly depends on the adopted value of the significance level, as well as statistical method applied. The percentage is somewhere between 70 and 30, but they advocated that the number is less then 50 (Flin \& Krywult 2002). In connection with the detection of substructures even in so-called relaxed clusters (A 85, A 1795, A 2199) we conclude that (almost) all clusters contain substructures. The question is not whether clusters do contain substructures, or not but what is the degree of substructure in a given cluster. Therefore, the existence of substructures

Send offprint requests to: $\mathrm{W}$. Godłowski, e-mail: godlows@oa.uj.edu.pl in clusters has to be considered as an essential property of these objects.

In a previous paper (Godłowski et al. 1998) we used a new method of searching for subclustering in galaxy clusters, based on the study of the alignment of galaxy planes. The theoretical simulations of the evolution of cosmic structures in the case of various scenarios predict different distributions of the angular momentum of galaxies, i.e. of galaxy orientations, which are represented as different forms of galaxy alignment within such structures (clusters and superclusters).

In the framework of the major classical evolutionary scenarios (Shandarin 1974a,b; Wesson 1982; Silk \& Efstathiou 1983) - where galaxy clusters are formed by random clumping of uniformly distributed galaxies - we expect to find a random distribution of rotation axes. This situation is predicted for example in the hierarchical clustering picture of Dekel (1985).

On the other hand, we expect to find coherence in the alignments of galaxy orientations in the case of the fragmentation scenarios. There are two main theories for the latter picture. Firstly, according to Ozernoy (1978) and Efstathiou \& Silk (1983), the turbulence model will lead to a situation where the rotation axes of the galaxies tend to be perpendicular to the major plane of the large-scale structure (supercluster). Galaxy planes and the planes of the superclusters should be aligned coherently. In the second model (Shandarin 1974a,b; Doroshkevich et al. 1978) - where the galaxies originate from adiabatic fluctuations - there occurs an alignment between rotation axes and the orientations of the corresponding 
larger cosmic structures in which galaxy planes and the planes of the superclusters are arranged perpendicular to each other. A third possibility - the recently proposed hedgehog model (MacGillivray et al. 1982b) - predicts a tendency for galaxy rotation axes to be orientated towards the centre of a supercluster.

Thus, the study of galaxy orientations has the potential to yield important information with respect to the formation and evolution of cosmic structures. Unfortunately, the practical applicability of tests of searching for galaxy alignments is difficult due to the observational problems and line-of-sight superpositions of galaxies separated over large distances.

Results from most of the previous studies (Thompson 1976, 1981; Jaaniste \& Saar 1978; Adams et al. 1980; Gregory 1981; MacGillivray 1982a,b; MacGillivray \& Dodd 1985; Flin \& Godłowsli 1986; Godłowski 1993; Flin 1995; Parnowski et al. 1994) have shown that there are coherent alignments of galaxy orientations in some clusters and superclusters. This finding was also confirmed in our previous paper (Godłowski et al. 1998). However, in some papers e.g. Bukhari (1998) no alignment of galaxies in clusters was found. Some authors (for example, Trevese et al. 1992) searched for the alignment between galaxy orientations and the connecting lines with the cluster center, did not find any. However, these authors, as well as van Kampen \& Rhee (1990), found a strong alignment between the first ranked galaxies and the major axes of the clusters.

The question of whether the alignments seen within larger structures are due to global effects connected with the supercluster as a whole or to a conglomeration of galaxy alignments from individual constituent subunits (i.e. clusters) needs to be addressed. This question has been, in the main, neglected. However, recently a series of papers (MacGillivray 1982a,b; Struble 1987, 1990; van Kampen 1990; Trevese et al. 1992; Han et al. 1995; Salvator-Sole \& Solanes 1993) has gone some way towards furthering our understanding in this area.

In this regard, the investigation of galaxy orientations in subclusters of rich galaxy systems may also provide further important information, especially concerning cluster evolution. This paper is the second one to date to deal with the question of possible alignments of galaxy planes within clusters with obvious subclustering.

\section{Method of the orientation analysis}

Up to now there have been two main methods for studying the orientations of galaxy rotation axes. The first one (Hawley \& Peebles 1975; H-P) undertakes the analysis of the observed position angles of the major axes of galaxy images. However, these angles can yield reliable information with respect to galaxy planes only for galaxies seen edge-on. Consequently, all the galaxies seen face-on or nearly face-on are excluded from this analysis. Also for such (face-on) galaxies could occur problem of correct determination of the position angle during measurement.

The other approach, proposed by Jaaniste \& Saar (1978; $\mathrm{J}-\mathrm{S}$ ), takes into account not only the position angles of galaxies but also the inclinations of the galaxy planes relative to the observer's line of sight. This inclination, however, is not defined clearly; it represents the orientation of the normal vector to one of the two possible orientations of the plane of the galaxy under consideration. Flin \& Godłowski (1986) corrected and improved the method of Jaaniste \& Saar (1978) eliminating some inconsistencies, and developed a method for analysing the distribution of these normal vectors. With the help of the latter approach, for such an investigation it is possible to use galaxies of any orientation, - including also face-on galaxies. Flin \& Godłowski's method allows one to compute two angles to describe the orientation of the normal to the galaxy plane (which is mostly accepted to be the rotation axis) with respect to the plane of the cluster or supercluster: 1) The angle $\delta_{D}$ between the normal to the galaxy plane and the plane of the cluster or supercluster and 2) the angle $\eta$ between the projection of the normal to the galaxy plane on to the plane of the corresponding cluster- or supercluster structure and the direction toward the structure centre. Finally, it is possible to analyse the distributions of these angles with the help of statistical methods in order to find any non-random trends. One should note, however, that the practical application of Flin \& Godłowski's method is not easy and requires a special procedure so as to be free of selection effects (Flin \& Godłowski 1986 Sect. 6.1; Godłowski 1993; Aryal \& Saurer 2000).

We should mention that the computation of the rotation axes of galaxies is not so easy in our case. In the ideal case, it requires (Godłowski 1993, 1994) the knowledge of the position angle $p$ and the inclination angle $i$ for particular galaxies. We can determine the inclination angle $i$ from the axial ratio $q$ of galaxies in the case of known true axial ratios (for particular galaxies). This is possible if we know the morphological types of these galaxies. Unfortunately, this is not the case in our investigation. In that situation we have to make an assumption as to the axial ratio. We assume that galaxies are oblate spheroids with a flatness ratio of some standard value (e.g. $q_{0}=0.2$ ). Moreover, it is known that elliptical galaxies have only a small angular momentum (if any). So in the case of elliptical galaxies it is more usefully so speak about the distribution of the normals to galaxy planes than about the distribution of the rotation axes. The above-mentioned problems with the reproduction of galaxy orientation from the geometric shape of the images are the reason for our concentration on the analysis of the position angles while analysing the aligment of galaxies.

In principle, it is possible to examine the orientations of galaxies (i.e. the angular momenta) with respect to different coordinate systems (e.g. the coordinate systems defined by the parent entity). In previous papers, Godłowski (1992, 1993) analysed the distribution of angular momenta of galaxies with respect to the plane of the local supercluster (LSC). This reflects the situation referred to above where the alignment of galaxy orientations in a supercluster is determined by the general structure of the supercluster. However, there is no reason a priori not to consider smaller structures (e.g. clusters or subclusters) as being the parent system.

In such a situation we would expect that galaxy alignments in clusters are connected to the main plane of the cluster (or subcluster) rather then that of the supercluster. Therefore, in principle we should consider other possibilities and look for the presence of different alignments of galaxy orientations in clusters with respect to different main planes. Bimodal distributions 
of orientations, as found by Gregory et al. (1981) (if detected) could support the existence of substructures. On the other hand, the absence of such bimodal or multimodal distributions cannot exclude the existence of subclusters of galaxies definitely.

As a first step, we transformed the coordinates and position angles $p$ of galaxies to a new coordinate system based on the cluster's (or subcluster) main plane (see Godłowski 1993 for a detailed description). The position angle of the cluster is determined in a straightforward way by the least-squares method, taking the coordinates of the galaxies as variables. We fitted the orthogonal regression line based on the minimum distance of the observed points from the regression line. In such a situation, the angle between the direction to the north pole of the coordinate system and the regression line is the position angle of the cluster.

The computation of the axial ratio $q$ and inclination angle (or pole direction) is not straightforward. Ideally, the method requires a fit of the cluster reference plane for two cluster poles derived by assuming it to be an oblate spheroid with flatness ratio of some standard value (e.g. $q=0.2$ ). One should remember that such an approximation is acceptable for galaxies even if we do not have any information about the morphological type of a particular galaxy. However, the application of such a procedure to clusters of galaxies could introduce large errors (we do not have sufficient knowledge yet of the true shapes of rich clusters). Therefore, this method can be used as a crude approximation only.

Alternatively, if we know the distance to the galaxies, we could fit their distribution in 3-D space with a covariance ellipse. Then, from the ellipse parameters we can determine the axial ratio of the cluster $q$, or even directly the spatial direction of the three axes of the ellipse. However, such an investigation is not possible in our case because we do not know the true distances of particular galaxies.

In the face of these difficulties, the approach we have adopted in our work is to assume that clusters are oblate spheroids and to vary the possible pole direction of the cluster around the whole celestial sphere. Subsequently, we look for possible alignments in all artificial coordinate systems.

In our approach, for a detailed analysis of galaxy alignment inside clusters we use the statistical parameter provided by the Fourier test, $\Delta_{11}$, (Flin \& Godłowski 1986; Godłowski 1993, 1994). It describes the preferential orientation of galaxy axes with respect to the cluster reference frame. For all clusters and subclusters under examination, we mapped the ratio of $\Delta_{11}$ divided by its formal error $\sigma\left(\Delta_{11}\right)$ as the cluster pole direction is moved around the entire celestial sphere. The resulting maps are analysed for correlations of the $\Delta_{11} / \sigma\left(\Delta_{11}\right)$ extreme values (maxima or minima) with the direction from the cluster centre to the derived cluster poles, and our line of sight. However, the interpretation is confused by galaxy superpositions along the line of sight. According to Godłowski \& Ostrowski (1996), such effects mask the existing preferential orientations of galaxies in the cluster to a high degree. Nonetheless, the comparison of such maps can give us important information concerning any possible subclustering. Most importantly, we may expect different directions of alignments in the presence of various subclusters. Hence, in our map we

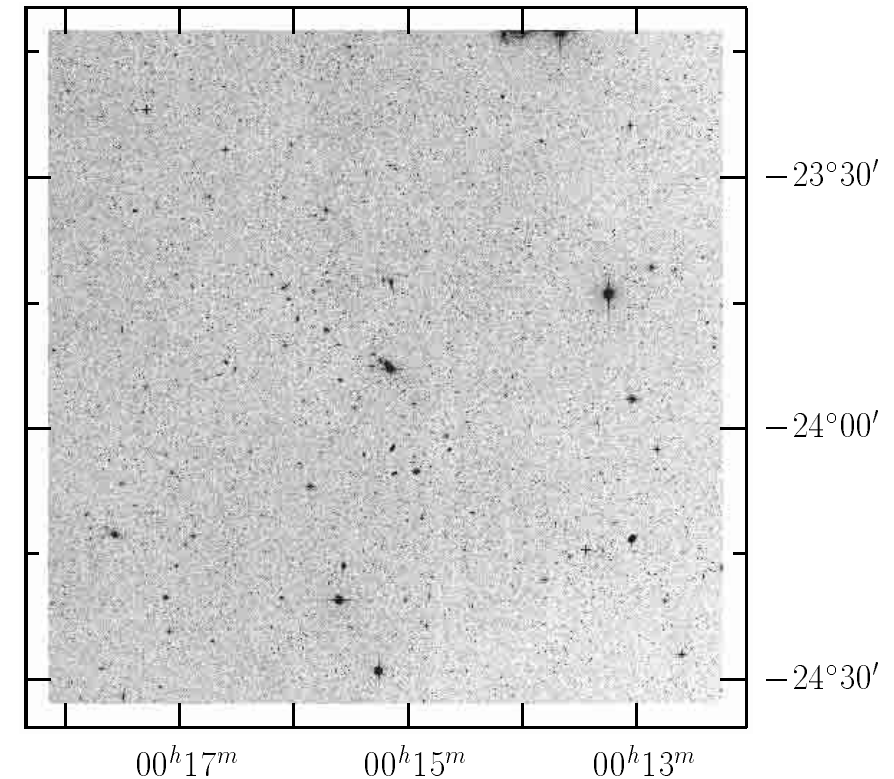

Fig. 1. Field of the Abell 14 galaxy cluster.

should find compact structures for possible maxima of the $\Delta_{11} / \sigma\left(\Delta_{11}\right)$ values. The appearence of such structures is an important indicator for the existence of subclustering.

\section{General description of the cluster Abell 14}

The galaxy cluster Abell 14 (Fig. 1) of redshift $z=0.0640$ (according to Dressler 1980) is of the structure type BM III according to Bautz \& Morgan (1970), and is a core-halo cluster according to Struble \& Rood (1987). Its richness class is $R=0$. Geller $\&$ Beers (1982) found a bimodal distribution of galaxies. West (1990) discovered only marginal substructures. Krywult et al. (1999) did not find evidence of subclustering. The central dominant galaxy is an elliptical galaxy according to Struble \& Rood (1987) or a cD-galaxy according to Andernach (1993). The orientation of the the central dominant galaxy is $\Theta_{\text {orien }}=51^{\circ}-53^{\circ}$ according to Struble \& Peebles (1985) and Binggeli (1982) respectively. Struble \& Peebles (1985), Dressler \& Shectman (1988b) and West (1989) found values cluster orientation to be between $\Theta_{\text {orien }}=51^{\circ}$ and $\Theta_{\text {orien }}=58^{\circ}$. With these values there seems to exist an alignment between the cluster and the dominant galaxy. The galaxy distribution within this cluster was investigated by Geller \& Beers (1982) and by Kriessler \& Beers (1987). The cluster shows a multiple structure according to both these investigations. There is a main concentration with an offset of roughly 5 arcmin between the position of the dominant galaxy and the number-density maximum of the galaxy distribution. Also, there are two further galaxy concentrations northeast and southwest of the main concentration. The combination of these 3 main groups generates the impression of an elongated cluster with the abovegiven orientation values. In addition, Geller \& Beers (1982) as well as Kriessler \& Beers (1987) found northwestern and southeastern extensions of the central group. All these irregularities of the galaxy distribution are hints of a complex cluster structure. This assumption is confirmed by the result of our 


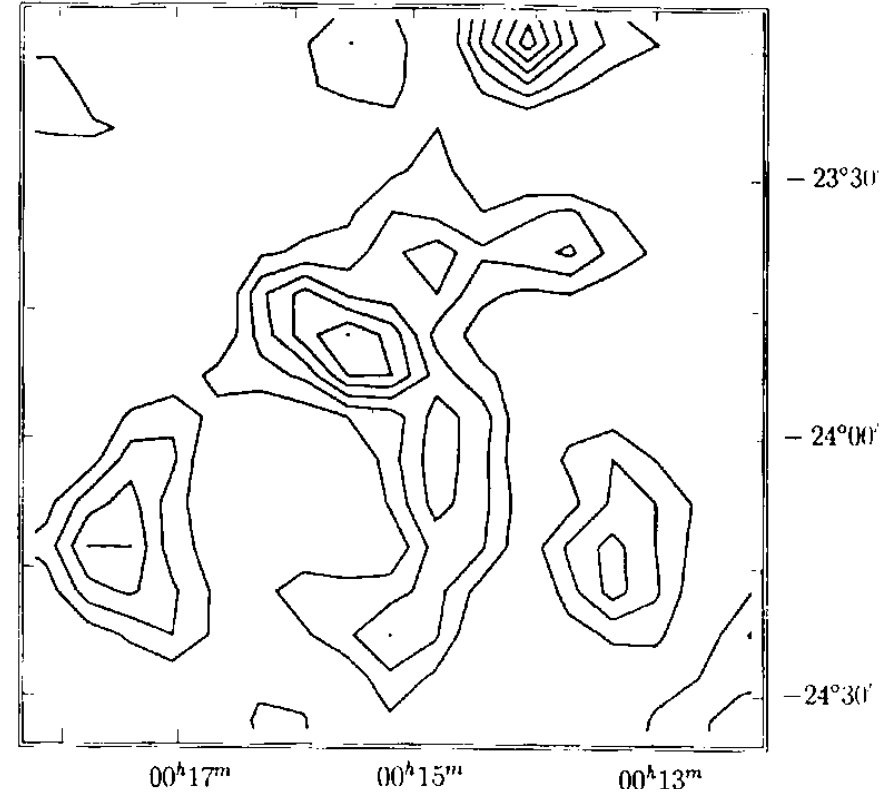

Fig. 2. Galaxy distribution in cluster field determined from Edinburgh-catalog data by Baier (1997) Baier \& MacGillivray (2003).

investigations. The values derived for cluster orientation correspond approximately to the orientation of the central cluster region shown in Fig. 2 according to our investigations (Baier 1997; Baier \& MacGillivray 2003) and in Fig. 3 according to the investigation of Geller \& Beers (1982). But these figures show additional structures in the outer region of the cluster. The investigation of galaxy distribution with the help of the Voronoi-tesselation (Voronoi 1908; Ikeuchi \& Turner 1991) provided an even more complicated structure picture (Fig. 4) (Baier 1997; Baier et al. 2003). The dominant galaxy is connected to the central group. Unlike appearances, most galaxies are arranged in single groups along filaments which originate in the cluster centre near the position of the dominant galaxy. This gives the impression of a spiral structure. Thus, the cluster is an example of a situation where the description of the cluster by radial number-density distribution or the estimation of a cluster ellipticity in connection with any equilibrium state (West 1990 and Struble \& Ftaclas 1994 found $\epsilon=0.34$ and $\epsilon=0.36$, respectively) seem to have limited applicability.

The complex structure, both in this case and for other examples, should rather force us to consider galaxy clusters from a new point of view in connection with the concept of Omarov (1995), who characterized such objects as nonequilibrium systems, forming a certain hierarchical level in the self-organizing universe according to Saslaw (1987) and Ostriker (1978). The justification for the assumption of nonequilibrium for most galaxy clusters follows from a large number of observational results (optical and X-ray substructures, temperature variations in gas, $\beta$-problem).

According to Nicolis (1986), any self-organizing system has a hierarchical structure. This discussion is consistent with the concept of multifractal matter distribution and self-similarity over a wide range of scales, as considered by Pietronero (1987) and others; i.e., a structure element of rank $l$ consists of analogous structures of rank $l-1$ (substructures).

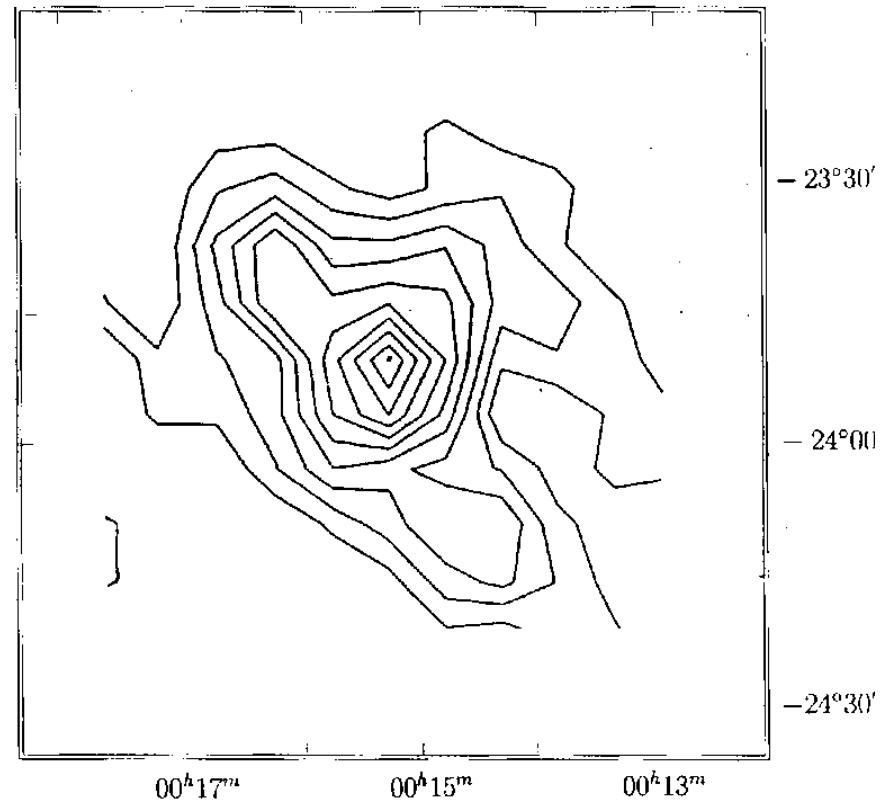

Fig. 3. Galaxy distribution determined in the field of Abell 0014 cluster by Geller \& Beers 1982.

Further details are reported by Dressler (1980) and Reuter \& Andernach (1990). Dressler (1980) measured the optical positions of the 79 brightest galaxies and determined their morphological classification. Reuter \& Andernach (1990) presented a radio map for the Abell 14 cluster obtained with the 100-m Effelsberg telescope. The dominant galaxy was not detected in the radio emission, either by them or by Mills et al. (1960) or by Mills \& Hoskins (1977). Reuter \& Andernach (1990) found four radio sources. Their sources 1-3 seem to be connected to the three galaxy groups in the region to the north of the dominant galaxy.

The X-ray emission of the unrelaxed cluster Abell 14 is very weak: $\log L_{x} \leq 44.13_{-0.48}^{+0.22}(2-10 \mathrm{keV})$ (McKee et al. 1980), $\log L_{x}=43.62(2-6 \mathrm{keV})$ (Johnson et al. 1983; Kowalski et al. 1984), $\log L_{x}<44.03$ (0.1-2.4 keV) (Ebeling 1993). Because of its low X-ray luminosity the Abell 14 cluster is comparable with clusters Abell 569 and Abell 2666 discussed by Baier et al. (1996). All of the three clusters should be characterized as poor clusters. They possess a weak gravitational potential only. The X-ray emission comes from the dominant galaxy only.

\section{Remarks pertaining to cluster evolution}

The characterization of Abell 14 as a poor cluster without a strong X-ray emission and consequently without any remarkable cooling flow excludes the formation of the Central Dominant Galaxy (CDG) by such a cooling flow. Rather we have to accept the alternative possibility, i.e. its formation by galactic cannibalism (Ostriker \& Tremaine 1975) which is expected when phenomena like dynamical friction, tidal stripping, and mergers are common. The formation of such central giant galaxies is easily obtained in N-body simulations of groups and clusters (e.g. Barnes 1989; Bode et al. 1994; 


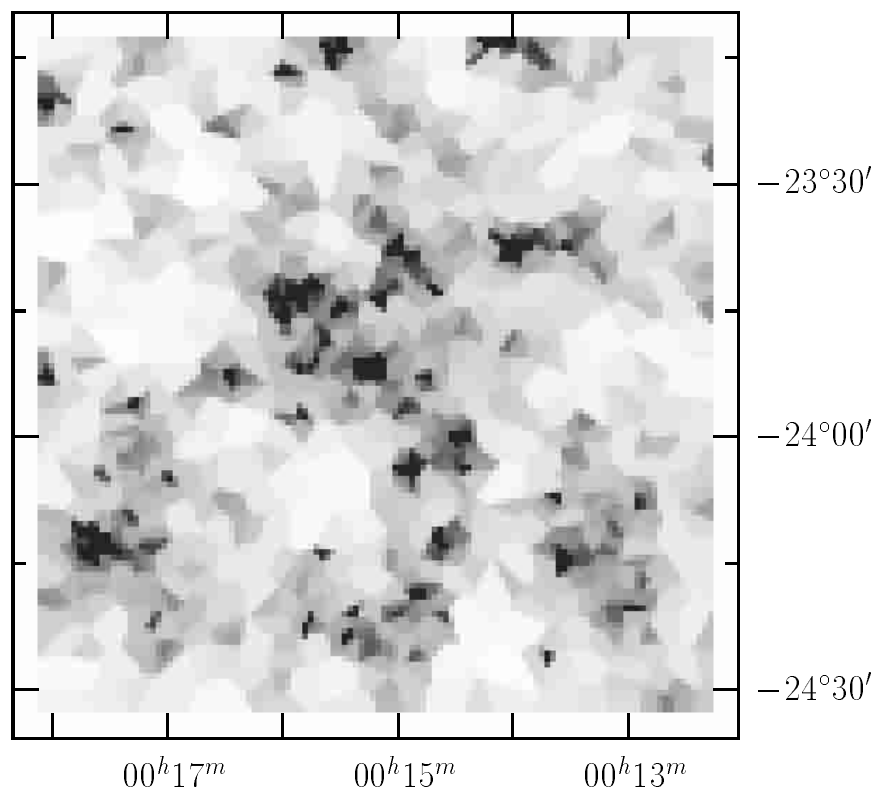

Fig. 4. Galaxy distribution in the field of Abell 14 cluster determined from Edinburgh-catalog data by Baier (1997), Baier et al. (2003) (Voronoi-tesselation).

Lima Neto \& Baier 1997). During the simulations of poor clusters by Lima Neto \& Baier even substructures appeared.

As mentioned above, according to West (1989), Zucca et al. (1993) and Einasto et al. (1994) the Abell 14 cluster is a member of a supercluster. The other poor clusters Abell 74, Abell 76, Abell 86, Abell 102, Abell 133 and Abell 151, as well as the rich clusters Abell 85 and Abell 119, belong to this supercluster, too. The latter two clusters contain cooling flows. We cannot exclude the possibility of encounters between these clusters and a following merging one. Therefore, the future formation of a structure similar to that in the case of Abell 754 (Baier et al. 1996; Godłowski et al. 1998) is possible. In this connection, we should consider such clusters as Abell 14 to be stepping stones for the formation of larger structures on the basis of the bottom-up scenario for the evolution of cosmic structures.

\section{Analysis of galaxy orientations}

We have analysed a sample of 974 galaxies in the cluster field. The data were taken from the COSMOS/UKST Southern Sky Object Catalogue (Yentis et al. 1991), which is a catalogue of stars and galaxies derived from glass copies of the Southern Sky SERC J Sky Survey. Initially, all galaxies down to a limiting magnitude of $B=20.0$ mag were selected. Subsequently, however, in order to reduce the contamination of our results by foreground and background objects, we eliminated galaxies outside the a region selected in line with our discussion of Sect. 3 on the basis of galaxy surface density and Voronoi tesselation. The galaxies retained for our analysis are shown in Fig. 5.

The aim of our investigations has been to compare the existence of galaxy alignments with the existence of cluster substructures. As before in our investigation of galaxy

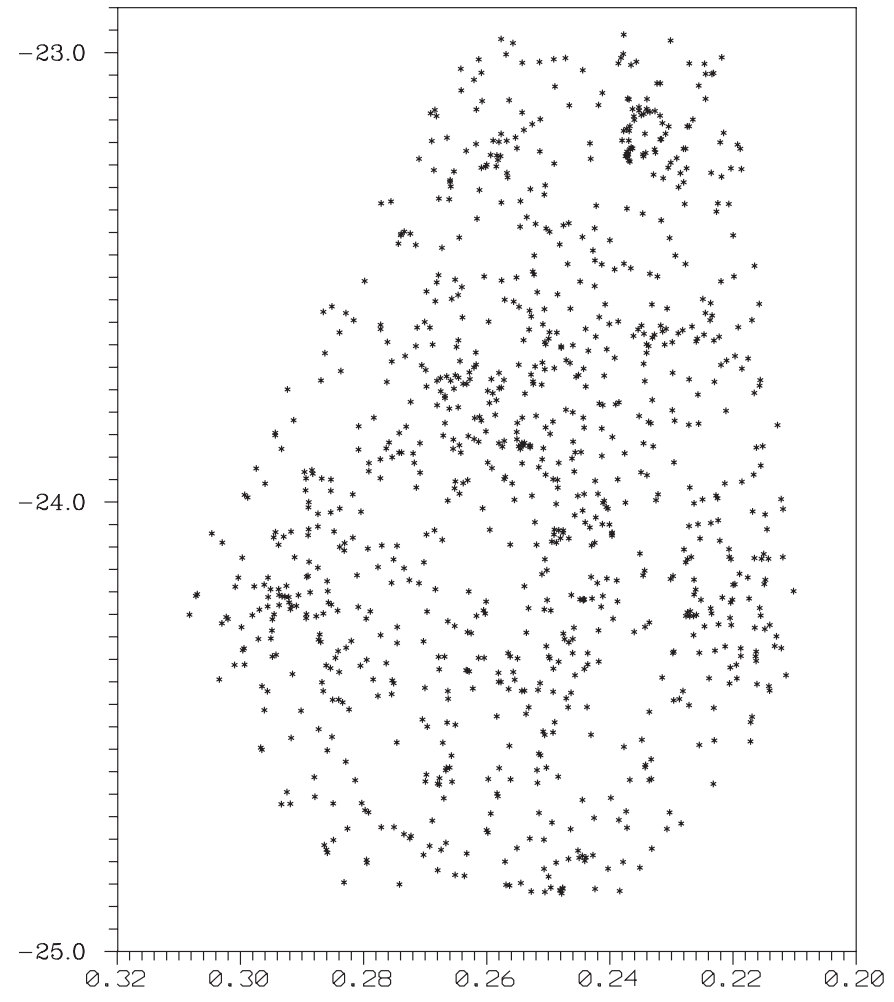

Fig. 5. Distribution of all galaxies in the cluster field from Edinburghcatalog data as function of $\alpha$ (in (hours) in decimal notation) and $\delta$ in (deg) for the epoch 2000.0.

alignments we used two methods. Firstly, we discussed the distribution of the position angles of galaxies. Secondly, we applied a method which used both position angles and inclinations. We found rather good evidence for alignment, and corresponding evidence for subclustering within the Abell 14 cluster.

In order to carry out our investigations the cluster area was split into different parts according to the constituent subcomponents with the help of our investigation of Sect. 3 (Figs. 2-4). At first glance, galaxy distribution in the cluster region is characterized by the central spiral structure (component 1) seen in Figs. 2 and 4, and by at least three surrounding concentrations A, B, and C (see below) of galaxies (component 2) (Fig. 6). Therefore, we divided the whole area into an inner region and an outer region according to the density maps. Figure 6 shows the border between the inner (substructure 1) and the outer (substructure 2) region and outer border of the outer region. The center of the inner structure (structure 1 in Table 1) is at $\alpha=00^{\mathrm{h}} 15^{\mathrm{m}} 45^{\mathrm{s}}, \delta=-23^{\circ} 50^{\prime}$. The outer region (component 2 in Table 1) contains, among others, three pronounced galaxy concentrations with centers at $\alpha=00^{\mathrm{h}} 17^{\mathrm{m}} 40^{\mathrm{s}}$, $\delta=-24^{\circ} 12^{\prime}$ (structure A), $\alpha=00^{\mathrm{h}} 13^{\mathrm{m}} 20^{\mathrm{s}}, \delta=-24^{\circ} 15^{\prime}$ (structure B) and $\alpha=00^{\mathrm{h}} 14^{\mathrm{m}} 00^{\mathrm{s}}, \delta=-23^{\circ} 15^{\prime}$ (structure C). It is called structure 2 (see Table 1). In all three cases (the cluster galaxy distribution in the inner region (structure 1), the galaxy distribution in the outer region (structure 2) and in the whole investigated region) we obtained the result that the position angles of the galaxy distributions are very close to $\Theta \sim 170^{\circ}$. However, because of the complicated cluster 


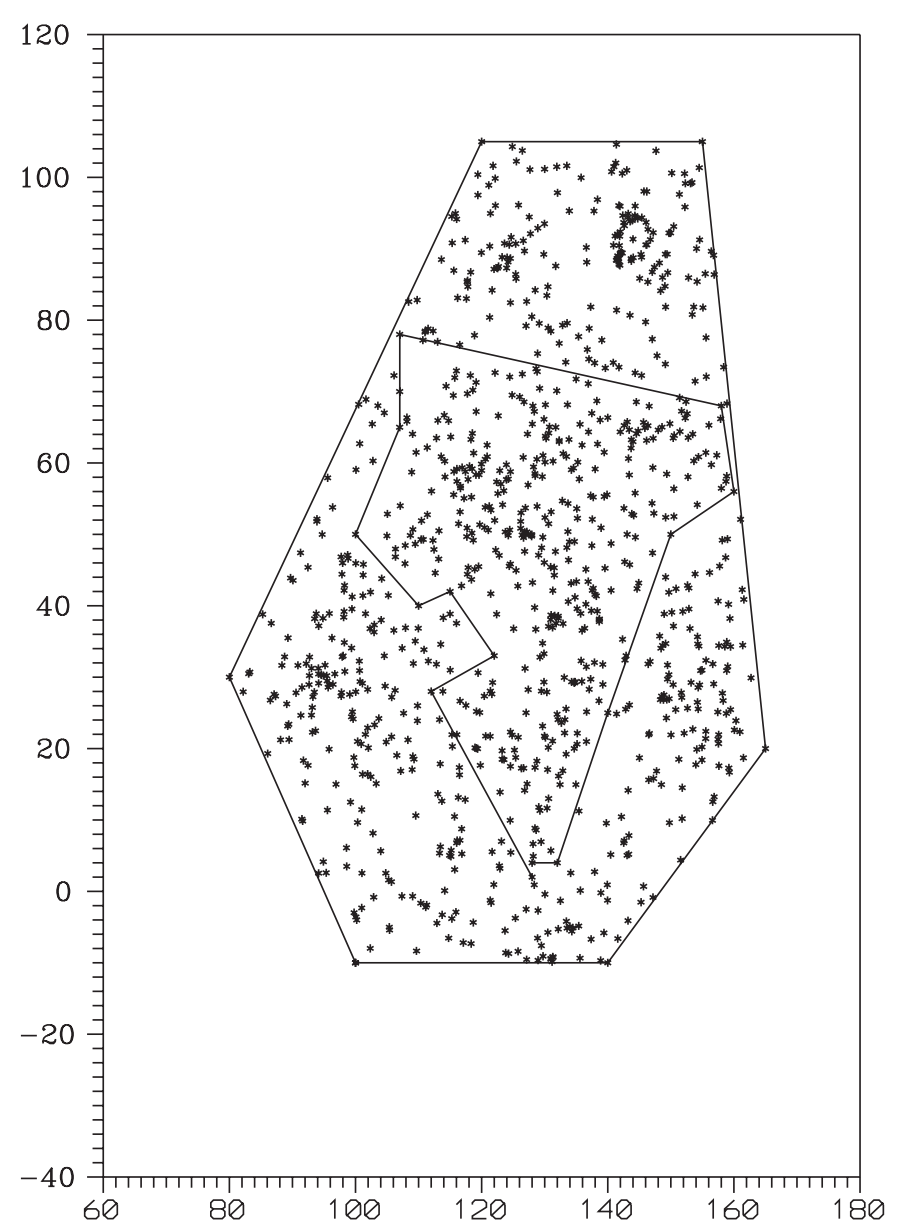

Fig. 6. Distribution of all galaxies in A14 cluster field from Edinburghcatalog data (in plate coordinates), showing inner and outer region borders.

structure there remains the question of the physical meaning of this agreement Also, there is a pronounced difference between the position angle of $\Theta \sim 170^{\circ}$ and the values derived in some previous papers by Struble \& Peebles (1985), Dressler $\&$ Shectman (1988) and West (1989). This difference occurs probably because of the very complex cluster structure. We analysed the whole inner region of the Abell 14 cluster. The orientation $\Theta \sim 55^{\circ}$ of the central part of the inner region (substructure $\mathrm{CP}$ ) centered at $\alpha=00^{\mathrm{h}} 15^{\mathrm{m}} 45^{\mathrm{s}}, \delta=-23^{\circ} 50^{\prime}$ agrees very well with the results of the above mentioned papers. All small substructures mentioned above are presented in Fig. 7.

The orientations of structures $\mathrm{A}, \mathrm{B}$ and $\mathrm{C}$ are $\Theta \sim 33^{\circ}$, $\Theta \sim 178^{\circ}$ and $\Theta \sim 37^{\circ}$ respectively. The results are not very different because $\Theta=178^{\circ}=-2^{\circ}\left(\Theta \in\left[0^{\circ} ; 180^{\circ}\right)\right)$. The similarity of the orientations of these three structures in the outer circular region was the reason why we decided to analyse the whole outer region, considering these structures $\mathrm{A}, \mathrm{B}$, and $\mathrm{C}$ separately.

We analysed the distribution of the position angles of 585 galaxies with axial ratios smaller than 0.75 . This means that our analysis of the position angles excluded the faceon galaxies because their position angles (for such galaxies) give no information connected with their angular momentum. Also for such (face-on) galaxies the problem of the correct

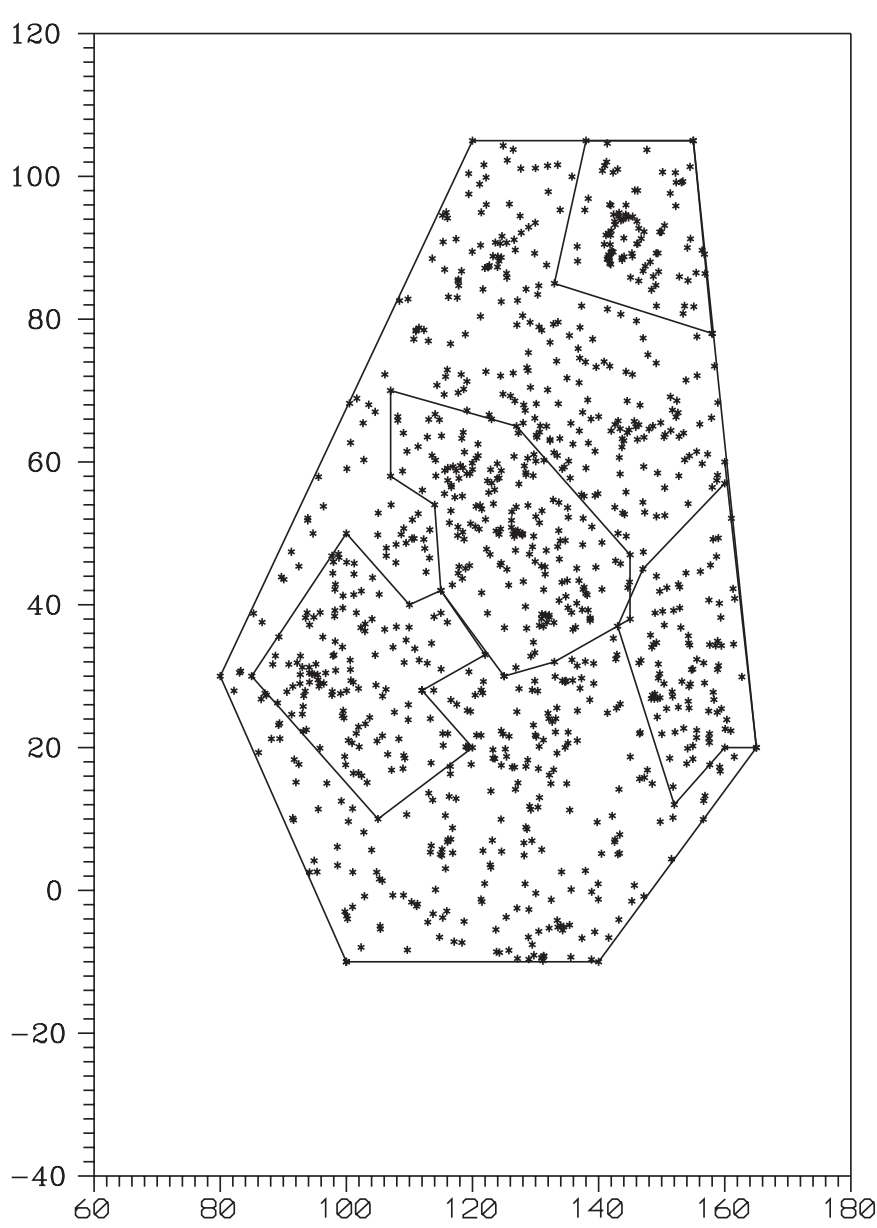

Fig. 7. Distribution of all galaxies in A14 cluster field from Edinburghcatalog data (in plate coordinates), showing small substructures borders.

determination of the position angle during measurement could occur. The results are given in Table 1 and Fig. 8. It should be pointed out that we do this analysis in the equatorial system because of the problems noted in Sect. 2 with the determination of the true pole direction of the cluster (i.e., we analysed the distribution of the position angles of galaxies $p$ in the equatorial system). The most important result is very strong alignment of the position angles in all regions investigated. It should be pointed out that it is not clear if the whole outer region - structure 2 in the Table 1 and/or substructures A, B, C belong to Abell 14, or if it is for example a cluster not belonging to the Abell 14 or possibly (substructure C) false detections occuring around a bright object. For this reason we analysed the whole cluster region, and seperately tested the inner and outer regions as the well as small substructures A, B, C and CP. We find that there is no differences between alignmenth of galaxies in the whole structure and in the substructures (also in the case of substructure C). From the three most important statistical tests used (see Appendix) we can reject the possibility of random distribution at a very high level. The maximum of this distribution of position angles is at $\theta \sim 30^{\circ}$ both for the whole cluster region (Fig. 8) and for the particular substructures. From this result we conclude that the galaxy position angles tend to be parallel 
Table 1. Test for isotropy in distribution of position angles $p$ for A 14 galaxies.

\begin{tabular}{rcrrrrrrrrrr}
\hline \hline & $\begin{array}{c}\text { Structure } \\
\text { number }\end{array}$ & $N$ & $\chi^{2}$ & $C$ & $P\left(\Delta_{1}\right)$ & $P\left(\Delta_{2}\right)$ & $\Delta_{11}$ & $\sigma$ & $W_{a}$ & $\sigma$ \\
\hline \multirow{2}{*}{$A 14$} & 0 & 585 & 112.3 & 69.03 & .000 & .047 & 0.308 & .058 & -.179 & .041 \\
& 1 & 233 & 59.2 & 18.85 & .000 & .283 & 0.302 & .093 & -.159 & .066 \\
& 2 & 352 & 87.6 & 50.14 & .000 & .140 & 0.312 & .075 & -.193 & .053 \\
& $\mathrm{~A}$ & 77 & 28.0 & 16.27 & .000 & .891 & 0.357 & .161 & -.208 & .114 \\
$\mathrm{~B}$ & 57 & 40.6 & 18.79 & .000 & .710 & 0.316 & .187 & -.281 & .132 \\
$\mathrm{C}$ & 62 & 29.2 & 5.35 & .000 & .577 & 0.180 & .180 & -.161 & .127 \\
& $\mathrm{CP}$ & 105 & 28.2 & -3.00 & .021 & .992 & 0.318 & .138 & -.210 & .098 \\
\hline
\end{tabular}

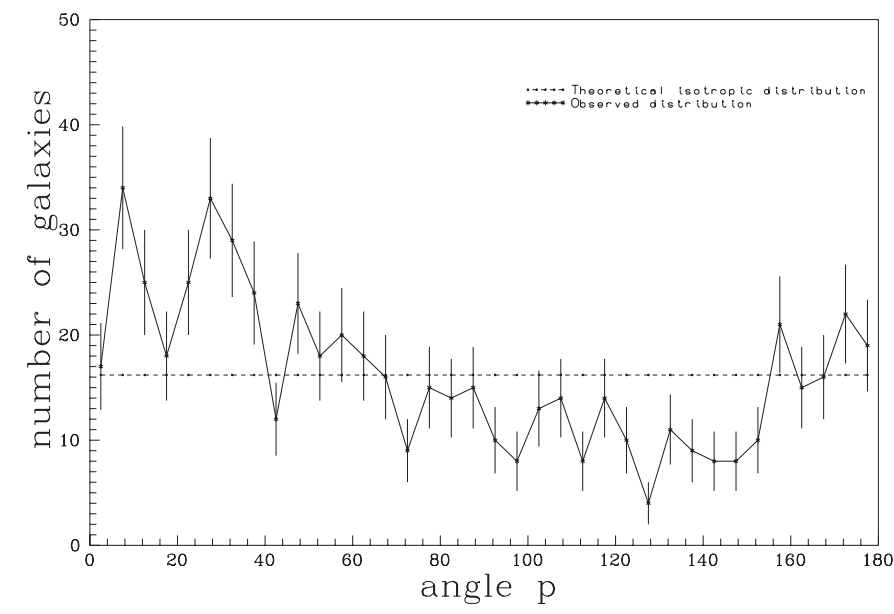

Fig. 8. Distribution of galaxy position angles in the whole cluster field.

to the cluster position angle (and also to the subcluster position angles). Further on the galaxy position angles are very close to the position angle of the CD galaxy. From this result (preferred position angle for edge-on galaxies close to the position angle of the whole cluster) we infer that galaxy planes are preferentially aligned parallel to the possible cluster plane (if one exists).

This result is similar to that obtained by Hawley \& Peebles (1976) but at a clearly higher level. However we should note that our result is different from those of some previous papers. The result found for the Local Supercluster by Janiste \& Saar (1978), Flin \& Godłowski (1986) and Godłowski (1993, 1994) (these authors did not consider the position angles but the orientations of the normals to galaxy planes) and confirmed by Parnowsky (1994) shows that galaxy planes are preferably oriented perpendicular to the Local Supercluster plane.

A similar result (i.e., the galaxy position angles being perpendicular to the position angle of the whole cluster) was found by Godłowski et al. (1998) during their analysis of the galaxy position angles in the Abell 754 cluster.

According to Di Fazio \& Flin (1988) such differences in alignments of the galaxy position angles (i.e. position angles of galaxies perpendicular or parallel to the the position angle of the whole cluster) could be connected to the existence of two cluster types - oblate and prolate. We cannot exclude the existence of different alignment - types in such different cluster types.
We cannot find any clear evidence for different shapes or different maxima of the distributions of the galaxy position angles in all regions considered hitherto. However, while investigating the alignment of the galaxy position angles we found another important result. From the Fourier test taking into account only the highest Fourier mode, i.e. when

$N_{k}=N_{0, k}\left(1+\Delta_{12} \cos 4 \theta_{k}+\Delta_{22} \sin 4 \theta_{k}\right)$

(see Godłowski 1994 for detailed explanation), we found a certain amount of anisotropy. This result could be an indication of a more complex bimodal distribution according to the discussion of Gregory et al. (1981). Such a bimodal distribution can be explained by the influence of various substructures. However, we were not able to find a definite proof of a correlation between substructures and different alignments. Thus, this interpretation should be treated with caution.

Next, we discuss the method originally proposed by Jaaniste \& Saar. There is a large difference between the number of galaxies in the whole cluster area and the number of galaxies with axial ratio smaller than 0.75 . We assume that there is an excess of face-on galaxies in our sample. Such a result can be due to our poor knowledge of the true galaxy shape for the majority of elliptical galaxies according to the discussion of Godłowski \& Ostrowski (1998). These authors found a similar effect in the data from the Tully-NGC Catalog (1988). Therefore, we should be very careful when discussing galaxy orientations reproduced from the geometric image shapes.

Despite all the above problems the results found by such methods could give us important information regarding the alignment of galaxies and possible subclustering (see below). We have analysed the distribution of angle $\delta_{D}$ giving information on the inclination of the normals to galaxy planes with respect to the cluster (coordinate system equator) plane. We have mapped the ratio of $\Delta_{11}$ divided by its formal error $\sigma\left(\Delta_{11}\right)$ for different possible cluster pole directions along the whole celestial sphere for the Abell 14 cluster, shown in Fig. 9. In the final map we have looked for any possible correlations between the extreme values of $\Delta_{11} / \sigma\left(\Delta_{11}\right)$ and different directions. There is a correlation between the maximum of this ratio and the direction of the line of sight to the cluster. This result confirms our previous assumption that the effect described above (Godłowski \& Ostrowski 1999) is present in our sample. According to us, the highly compact structure of the observed maximum is of special importance. This is possibly an 


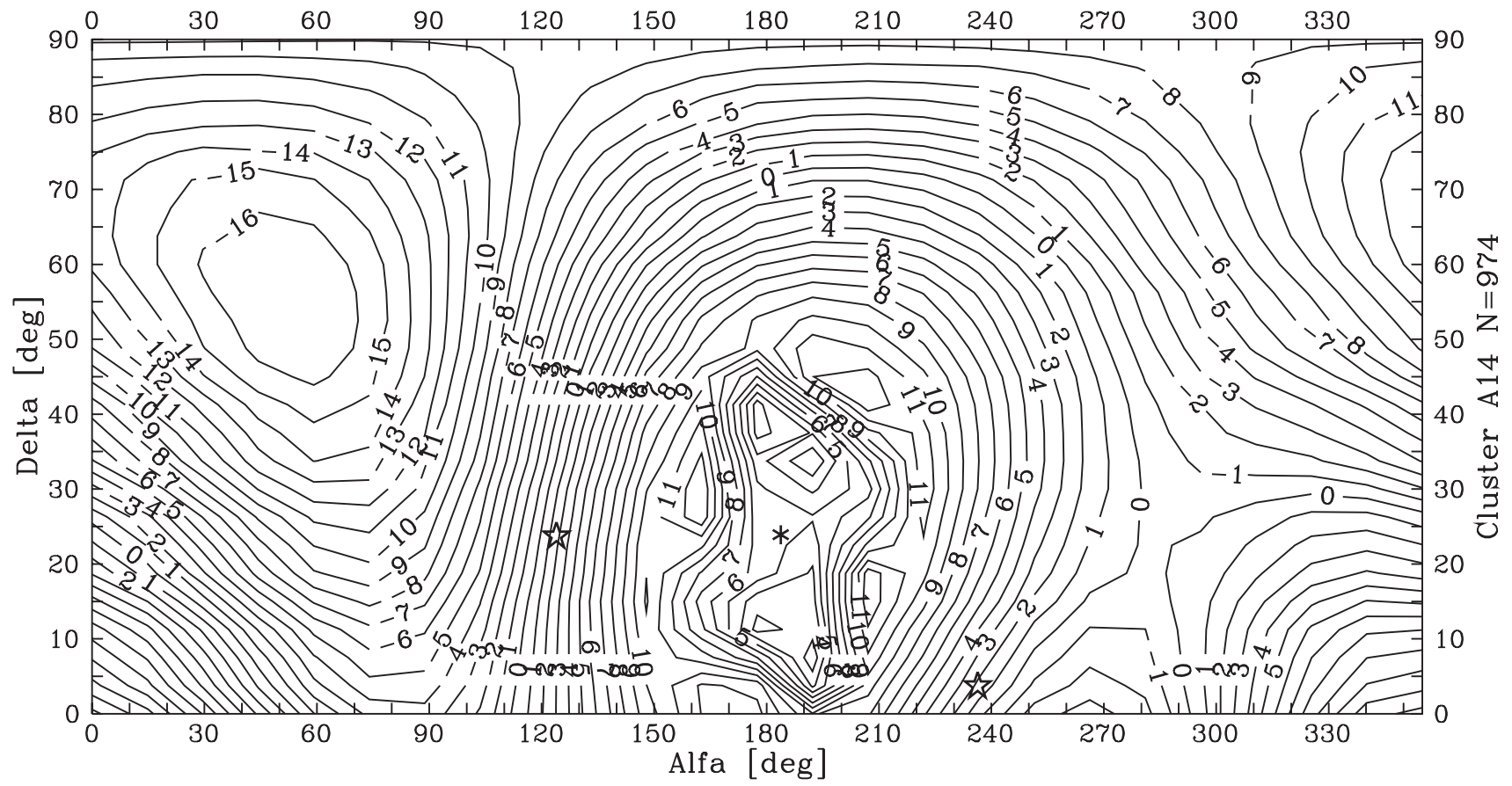

Fig. 9. Map of the distribution of $\Delta_{11} / \sigma\left(\Delta_{11}\right)$ (for $\delta_{D}$ ) angle presented in $\alpha, \delta$ coordinates. At the map we denoted the important directions as the line of sight (from the earth) marked with $\star$ and the possible direction of the cluster pole with the assumption of a true ellipticity of 0.2 marked with a big empty star.

indication of the existence of a substructure with a different kind of alignment.

In a rather small area of Fig. 9 we found a strong negative extremum. This result means that there is a very strong deficit of galaxies with normals to the galaxy plane pointing in that direction. It seems to us that the most probable explanation of such a phenomenon is the excess of edge-on galaxies with position angles $p \sim 30^{\circ}$. This is a clear confirmation of a real general alignment within this cluster.

Unfortunately, from our data we have no information regarding the morphological galaxy types. Therefore, we are unable to discuss the question of alignments according to different morphological types.

\section{Conclusion}

From the optical observations of the cluster we conclude the existence of substructures in Abell 14. The cluster is a poor cluster. The weak X-ray emission comes from the central dominant galaxy only.

We find strong evidence for the alignment of galaxy position angles in the cluster in the sense that the major planes of galaxies tend to be parallel to the direction of the position angle of the major axis of the cluster. Consequently, the normal to the galaxy planes (which is mostly accepted to be direction of angular momenta) are preferentially aligned perpendicular to the cluster plane. The direction of the alignment is perpendicular to that found by us in the case of Abell 754 (Godłowski et al. 1998). This could be a sign of the existence of different types of galaxy alignments in clusters. Moreover, from the analysis of the distribution of the galaxy positions angles we find some evidence for possible subclustering inside the cluster as a whole. This result is confirmed by the investigation of the distribution of the normals to the galaxy planes. Moreover, we can confirm the existence of the so-called lineof-sight effect discovered by Godłowski \& Ostrowski 1999 for galaxies belonging to the clusters in the Tully-NGC Catalogue (1988).

The similarity of the direction of the alignment of the galaxy position angles in the inner and outer regions, as well as in all structures, is an indication of a physical connection between them.

Acknowledgements. We thank the anonymous referee for remarks that help us improve the original version of the paper.

\section{Appendix A: Statistical methods applied}

In order to check the distribution of galaxy orientation angles $(\delta, \eta)$ and position angles $p$, we tested whether the respective distribution of the $\delta, \eta$ or $p$ angles is isotropic. We applied statistical tests originally introduced by Hawley \& Pebbles (1975) and Kindl (1987), and later modified by us (see the detailed description in Godłowski 1993, 1994). Below, a short summary of the tests considered here (not always explicitly) is presented: the $\chi^{2}$-test, the Fourier test and the auto-correlation test. In all of these tests, the entire range of the $\theta$ angle (where for $\theta$ one can put $\delta+\Pi / 2, \eta$ or $p$ respectively) is divided into $n$ bins, which gives in the $\chi^{2}$ test $(n-1)$ degrees of freedom. During the analysis of the whole cluster (i.e. the two main parts of the cluster region discussed above) we use $n=36$ bins of equal width (while during the analysis of the small subclusters we use $n=18$ bins because of the small numbers of galaxies). Let $N$ denote the total number of galaxies in the considered cluster, and $N_{k}$ - the number of galaxies with orientations 
within the $k$ th angular bin. Moreover, $N_{0}$ denotes the mean number of galaxies per bin, and finally, $N_{0, k}$ - the expected number of galaxies in the $k$ th bin. The $\chi^{2}$-test of the distribution yields the critical value of 49.8 for 35 degrees of freedom and 27.6 for 17 degrees of freedom (at the siginificance level $\alpha=0.05)$

$\chi^{2}=\sum_{k=1}^{n} \frac{\left(N_{k}-N_{0, k}\right)^{2}}{N_{0, k}}$.

This is minimised in model fitting, with the use of the modelprovided distribution $N_{0, k}$. However, when we consider individual subclusters, the number of galaxies involved is small in some cases, and the $\chi^{2}$ test will not neccessarily work well (e.g. the $\chi^{2}$ test requires the expected number of data per bin to equal at least 7; see, however, Snedecor \& Cochran 1967 and Domanski 1979). As a check, in a few cases we repeated the derivations for different values of $n$, but no significant differences appeared.

However, the main statistical test used in the present paper is the Fourier test. In the Fourier test the actual distribution $N_{k}$ is approximated as

$$
\begin{aligned}
N_{k}= & N_{0, k}\left(1+\Delta_{11} \cos 2 \theta_{k}+\Delta_{21} \sin 2 \theta_{k}\right. \\
& \left.+\Delta_{12} \cos 4 \theta_{k}+\Delta_{22} \sin 4 \theta_{k}\right) .
\end{aligned}
$$

If we take into account only the first and second Fourier modes separately, then we obtain the following expression for coefficients $\Delta_{i j}(i, j=1,2)$ :

$$
\begin{aligned}
& \Delta_{1 j}=\frac{\sum_{k=1}^{n}\left(N_{k}-N_{0, k}\right) \cos 2 J \theta_{k}}{\sum_{k=1}^{n} N_{0, k} \cos ^{2} 2 J \theta_{k}}, \\
& \Delta_{2 j}=\frac{\sum_{k=1}^{n}\left(N_{k}-N_{0, k}\right) \sin 2 J \theta_{k}}{\sum_{k=1}^{n} N_{0, k} \sin ^{2} 2 J \theta_{k}},
\end{aligned}
$$

with the standard deviation

$$
\begin{aligned}
& \sigma\left(\Delta_{11}\right)=\left(\sum_{k=1}^{n} N_{0, k} \cos ^{2} 2 \theta_{k}\right)^{-1 / 2} \approx\left(\frac{2}{n N_{0}}\right)^{1 / 2}, \\
& \sigma\left(\Delta_{21}\right)=\left(\sum_{k=1}^{n} N_{0, k} \sin ^{2} 2 \theta_{k}\right)^{-1 / 2} \approx\left(\frac{2}{n N_{0}}\right)^{1 / 2} .
\end{aligned}
$$

where $N_{0}$ is the average of all $N_{0, k}$. However, we should note that we could formally repleace the symbol $\approx$ with $=$ only in the cases where all $N_{0, k}$ are equal (for example when testing the isotropy of the distribution of the position angle). The probability that the amplitude

$\Delta_{j}=\left(\Delta_{1 j}^{2}+\Delta_{2 j}^{2}\right)^{1 / 2}$

is greater than a certain chosen value is given by the formula:

$P\left(>\Delta_{j}\right)=\exp \left(-\frac{n}{4} N_{0} \Delta_{j}^{2}\right)$,

while the standard deviation of this amplitude is

$\sigma\left(\Delta_{j}\right)=\left(\frac{2}{n N_{0}}\right)^{1 / 2}$.

From the value of $\Delta_{11}$ one can deduce the direction of the departure from isotropy. If $\Delta_{11}<0$, then, for $\theta \equiv \delta+\pi / 2$, excess of galaxies with rotation axes parallel to the cluster plane is present. For $\Delta_{11}>0$ the rotation axes tend to be perpendicular to the cluster plane. Similarly, while analysing the distribution of the galaxy position angles $(\theta \equiv p)$, if $\Delta_{11}<0$, an excess of galaxies with position angles parallel to the plane of the coordinate system (i.e. normal to galaxies perpendicular to the plane of the coordinate system) is present. For $\Delta_{11}>0$, the galaxy position angles are perpendicular to the plane of the coordinate system.

The auto-correlation test quantifies the correlations between galaxy numbers in adjoining angular bins. The correlation function is defined as

$C=\sum_{k=1}^{n} \frac{\left(N_{k}-N_{0, k}\right)\left(N_{k+1}-N_{0, k+1}\right)}{\left[N_{0, k} N_{0, k+1}\right]^{1 / 2}}$.

In the case of an isotropic distribution, we expect $C=0$ with the standard deviation:

$\sigma(C)=n^{1 / 2}$.

\section{References}

Andernach, H. 1993, private communication

Adams, M. T., Strom, K. M., \& Strom, S. E. 1980, ApJ, 238, 445

Aryal, B., \& Saurer, W. 2000, A\&A, 364, 197

Baier, F. W., \& Mai, W. 1978a, Astron. Nachr. 299, 69

Baier, F. W., \& Mai, W. 1978b, Astron. Nachr. 299, 197

Baier, F. W. 1979a, Astron. Nachr. 300, 85

Baier, F. W. 1979b, Astron. Nachr. 300, 133

Baier, F. W. 1979c, Astron. Nachr. 300, 243

Baier, F. W. 1980a, Astron. Nachr. 301, 17

Baier, F. W. 1980b, Astron. Nachr. 301, 165

Baier, F. W. 1983, Astron. Nachr. 304, 211

Baier, F. W. 1997, Thesis Universitaet Potsdam

Baier, F. W., Brunner, H., MacGillivray, H. T., \& Wipper, H., in preparation

Baier, F. W., Lima Neto, G. B., \& Wipper, H. 1996, Astron. Nachr, 317,77

Baier, F. W., \& MacGillivray, H. T., in preparation

Baier, F. W., \& Wipper, H. 1995, Astron. Nachr., 316, 319

Barnes, J. E. 1989, BAS, 21, 1170

Bautz, L. P., \& Morgan, W. W. 1970, ApJ, 162, L149

Bhukhari, F. A. 1998, Thesis, University of Sydney

Binggeli, B. 1982, A\&A, 107, 338

Bird, C. M. 1994, AJ, 107, 1637

Bode, P. W., Berrington, R. C., Cohn, H. N., \& Lugger, P. M. 1994, ApJ, 433, 479

Briel, U. G., Henry, J. P., \& Schwarz, R. A. 1991, A\&A, 246, L10

Dekel, A. 1985, ApJ, 298, 461

Domanski, C. 1979, Statystyczne testy nieparametryczne, PWE, Warszawa (in Polish)

di Fazio, A., \& Flin, P. 1988, A\&A, 200, 5

Doroshkevich, A. G., Saar, E. M., \& Shandarin, S. F. 1978, in ed. M. S. Longair, \& J. Einasto, The Large Scale Structure of the Universe (Dordrecht, D. Reidel), IAU Symp., 79, 423

Dressler, A. 1980, APJS, 42, 565

Dressler, A., \& Shectman, S. A. 1988, AJ, 95, 985

Ebeling, H. 1993, Thesis, MPE-Report 250

Efstathiou, G. A., \& Silk, J. 1983, The Formation of Galaxies, Fund. Cosmic Phys. 9, 1

Einasto, M., Einasto, J., Tago, E., \& Dalton, G. B. 1994, ESO Scientific Preprint no. 987 
Flin, P. 1995, Comments Astrophys., 18, 81

Flin, P., \& Godłowski, W. 1986, MNRAS, 222, 525

Flin, P., \& Krywult, J. 2000, in ed. A. Mazur, O. Le Fevre, \& V. Le Brun, ASP Conf. Ser., 200, 406

Flin, P., \& Krywult, J. 2002 in Tracing Cosmic Evolution with Galaxy Clusters, ed. S. Borgani, M. Mezzeti, \& R. Valdarnini, ASP Conf. Ser., 268, 365

Geller, M. J., \& Beers, T. C. 1982, PASP, 94, 421

Godłowski, W., 1992, in The Early Universe and Cosmic Structures, ed. C. Balkowsky (Gif-sur-Yvette: Éditions Frontières), , 435

Godłowski, W. 1993, MNRAS, 265, 874

Godłowski, W. 1994, MNRAS, 271, 19

Godłowski, W., Baier, F. W., \& MacGillivray, H. T. 1998, A\&A, 339, 709

Godłowski, W., \& Ostrowski, M. 1999, MNRAS, 303, 50

Gregory, S. A., Thompson, L. A., \& Tifft, W. G. 1981, ApJ, 243, 411

Han, C., Gould, A., \& Sackett, P. 1995, ApJ, 445, 46

Hawley, D. L., \& Peebles, P. J. E. 1975, AJ, 80,

Ikeuchi, S., \& Turner, E. 1991, MNRAS, 250, 519

Jaaniste, J., \& Saar, E. 1978, in The Large Scale Structure of the Universe (Dordrecht, D. Reidel), ed. M. S. Longair, \& J. Einasto, IAU Symp., 79, 448

Johnson, M. W., Cruddace, R. G., Ulmer, M. P., Kowalski, M. P., \& Wood, K. S. 1983, ApJ, 266, 425

Jones, C., \& Forman, W. 1990, Adv. Space Res., 10, 209

Jones, C., \& Forman, W. 1992, in Clusters and Superclusters of Galaxies, ed. A. C. Fabian (London: Kluwer), NATO ASI, 366, 49

Kindl 1987, AJ, 93, 1024

Kowalski, M. P., Ulmer, M. P., Cruddace, R. G., \& Wood, K. S. 1984, ApJS, 56, 403

Kriessler, J. R., \& Beers, T. C. 1987, AJ, 113, 80

Krywult, J., MacGillivray, H. T., \& Flin, P. 1999, A\&A, 351, 883

Lima Neto, G. B., \& Baier, F. W. 1997, A\&A, 320, 717

MacGillivray, H. T., Dodd, R. J., McNally, B. V., \& Corwin, Jr. H. G. 1982a, MNRAS, 198, 605

MacGillivray, H. T., Dodd, R. J., MaNally, B. V., Lightfoot, J. F., Corwin, Jr. H. G., \& Heathcote, S. R. 1982b, Ap\&SS, 81, 231

MacGillivary, H. T., \& Dodd, R. J. 1985, A\&A, 145, 269

McKee, J. D., Mushotzky, R. F., Boldt, E. A., et al. 1980, ApJ, 242, 843

Mills, B. Y., Slee, O. B., \& Hill, E. R. 1960, Aust. J. Phys., 13, 676

Mills, B. Y., \& Hoskins, D. G. 1977, Aust. J. Phys., 30, 509

Muriel, H., \& Lambas, D. G. 1992, AJ, 103, 393

Mohr, J. J., Evrard, A. E., Fabricant, D. G., \& Geller, M. J. 1995, ApJ, 447,8
Nicolis, J. C. 1986, Dynamics of Hierarchical Systems (BerlinHeidelberg: Springer Verlag)

Omarov, T. B. 1995, Astron. Astrophys. Trans., 7, 265

Ostriker, J. P. 1978, The large Scale Structure of the Universe, ed. M. S. Longair, \& J. Einasto (Reidel Publ.), IAU Symp., 63, 393

Ostriker, J. P., \& Tremaine, S. D. 1975, ApJ, 202, L113

Ozernoy, L. M. 1978, in The Large Scale Structure of the Universe (Dordrecht: D. Reidel), ed. M. S. Longair, \& J. Einasto, IAU Symp., 79, 427

Parnovsky, S. L., Karachentsev, I. D., \& Karachentseva, V. E. 1994, MNRAS, 268, 665

Pietronerow, L. 1987, Physica A, 144, 257

Reuter, H.-P., \& Andernach, H. 1990, A\&AS, 82, 279

Rhee, G., van Haarlem, M. P., \& Katgert, P. 1991, A\&A, 246, 301

Salvador-Sole, E., \& Solanes, J. M. 1993, ApJ, 417, 427

Saslaw, W. C. 1987, Gravitational Physics of Stellar and Galactic Systems (Cambridge: Cambridge University Press)

Silk, J., \& Efstathiou, G. A. 1983, Fund. Cosmic Phys., 9, 1

Shandarin, S. F. 1974a, Astron. Zh., 51, 667

Shandarin, S. F. 1974b, Sov. Astron., 18, 392

Snedecor, G. W., \& Cochran, W. G. 1967, Statistical Methods (Iowa Univ. Press)

Struble, M. F. 1987, ApJ, 317, 668

Struble, M. F. 1990, AJ, 99, 743

Struble, M. F., \& Ftaclas, C. 1994, AJ, 108, 1

Struble, M. F., \& Peebles, P. J. E. 1985 AJ, 90, 582

Struble, M. F., \& Rood, H. J. 1987, ApJS, 63, 555

Thompson, I. A. 1976, ApJ, 209, 22

Thompson, I. A. 1981, Mercury 10, 122

Trevese, D., Cirimele, G., \& Flin, P. 1992, AJ, 104, 935

Tully, R. B. 1984, AJ, 281, 31

Tully, R. B. 1988, Nearby Galaxies Catalog (Cambridge Univ. Press)

van Kampen, E., \& Rhee, G. F. R. N. 1990, A\&A, 237, 283

Voronoi, G. 1908, J. Reine angew. Math., 134, 198

Webster, R., Fitchett, M., Hewett, P., \& Colles, M. 1988, in ed. J. Audouze, M. C. Peletan, \& A. Szalay, Large Scale Structure of the Universe (Dordrecht: Kluwer Academic Publisher), IAU Symp., 130, 537

Wesson, P. S. 1982, Visitas Astron., 26, 225

West, M. J. 1989, ApJ, 347, 610

West, M. J., \& Bothun, G. D. 1990, ApJ, 350, 36

Yentis, D. J., Cruddace, R. G., Gursky, H., et al. 1991, in ed. H. T. MacGillivray, E. B. Thomson, Digitised Optical Sky Surveys (Dordrecht: Kluwer), 67

Zucca, E., Zamorani, G., Scaramella, R., \& Vettolani, G. 1993, ApJ, 407,470 